\title{
Inter-Set Voluntary Hyperventilation-Aided Recovery Does Not Improve Performance of Bench Press and Squat in Recreationally Trained Individuals
} Original Research

Jeffrey D. Buxton ${ }^{1}$, Philp J. Prins ${ }^{1}$, Edward J. Ryan ${ }^{3}$, Dalton W. Jones ${ }^{1}$, Isaac J. Thrasher ${ }^{1}$, Madison N. Faulkner ${ }^{1}$, Elaine M. Robertson ${ }^{1}$, Gary L. Welton ${ }^{2}$, Dana L. Ault ${ }^{1}$

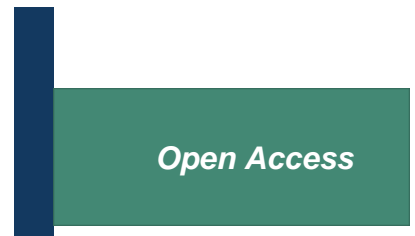

Published: January 26, 2022

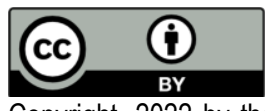

Copyright, 2022 by the authors. Published by Research Directs and the work is licensed under the Creative Commons Attribution 4.0 International License. To view a copy of this license, visit http://creativecommons .org/licenses/by/4.0/

Research Directs in Strength and Performance: 2022, Volume 2 (Issue 1): 2

ISSN: $2768-5187$
${ }^{1}$ Department of Exercise Science, Grove City College, Grove City, Pennsylvania, US A

${ }^{2}$ Department of Psychology, Grove City College, Grove City, Pennsylvania, USA

${ }^{3}$ Department of Exercise Science, Chatham University, Pittsburgh, Pennsylvania, USA

\begin{abstract}
Introduction: To examine the effects of voluntary hyperventilation $(\mathrm{VH})$ between sets of bench press (BP) and squat (SQ) at 70 and $90 \% 1 \mathrm{RM}$ on repetitions to failure, power, bar velocity, blood lactate, session RPE (sRPE), and muscle oxygen saturation $\left(\mathrm{SmO}_{2}\right)$.

Methods: Fifteen recreationally trained $(2.92 \pm 2.18$ yrs. of resistance training experience) college-aged males (20.27 \pm 1.39 yrs., $182.40 \pm 7.42$ $\mathrm{cm}, 82.23 \pm 10.84 \mathrm{~kg}$ ) performed three sets of BP and SQ to failure at 70 and $90 \% 1 \mathrm{RM}$ on separate days with normal breathing (CON) or $30 \mathrm{sec}$ of VH during inter-set rest periods.

Results: There were no significant differences between conditions for repetitions, power, velocity and sRPE ( $p$ 's $>0.05$ ) at either intensity. VH resulted in a slight attenuation of blood lactate accumulation between sets two and three of SQ $(p=0.037)$. There was a significant condition and intensity interaction for $\mathrm{SmO}_{2}$ of the pectoralis $(p=0.034)$ with $\mathrm{VH}$ producing higher $\mathrm{SmO} 2$ at $90 \%$ 1 $\mathrm{RM}$ and lower $\mathrm{SmO}_{2}$ at $70 \%$ 1 $\mathrm{RM}$ than the CON.

Conclusions: Voluntary hyperventilation did not produce an ergogenic effect in recreationally trained individuals which, when considering current evidence, suggests other factors including training experience, may influence the effectiveness of $\mathrm{VH}$.
\end{abstract}

Key Words: resistance training, respiratory alkalosis, breathwork

Corresponding author: Jeffrey D. Buxton, buxtonjd@gcc.edu

\section{Introduction}

A small but growing body of research has focused on the potential ergogenic effects of various breathwork practices including voluntary hyperventilation $(\mathrm{VH})$; an intentional and sustained increase in breathing frequency and/or depth leading to respiratory alkalosis and a reduction in hydrogen ions ${ }^{1-7}$. During exercise hydrogen ions build up and impair excitationcontraction-coupling resulting in reduced energy supply and fatigue ${ }^{7}$. The reduction in hydrogen ions from $\mathrm{VH}$ may therefore delay muscle fatigue and subsequently improve performance in activities relying on sustained muscular contractions ${ }^{4,6,7}$. Jacob et al. ${ }^{3}$ determined that VH performed prior to a $50 \mathrm{~m}$ swim resulted in significantly faster times. Additionally, Fujii et al. ${ }^{8}$ and Dobashi et al. ${ }^{9}$ found that 5 or $20 \mathrm{~min}$ of $\mathrm{VH}$ prior to Wingate sprints resulted in similar 
peak and mean power outputs but lower $\mathrm{O}_{2}$ uptake. Sakamoto and colleagues ${ }^{4}$ investigated the effect of $30 \mathrm{sec}$ of VH during the rest periods between repeat cycling sprints. There were no differences in peak or mean power outputs, however, the decrease in power from set to set was attenuated by VH ${ }^{4}$. A follow-up study by Sakamoto et al. ${ }^{6}$ investigated the use of a short $(15 \mathrm{sec})$ and long $(45 \mathrm{sec}) \mathrm{VH}$ protocol during the rest periods between sprints. Interestingly, neither protocol resulted in a conclusive ergogenic effect, suggesting that $30 \mathrm{sec}$ of $\mathrm{VH}$ may be the most ideal duration ${ }^{6}$.

Resistance training is another of form anaerobic exercise that may benefit from respiratory alkalosis induced by VH. In 2020, Sakamoto et al. ${ }^{7}$ found significant increases in repetitions to failure of leg press and bench press performed at $80 \% 1 \mathrm{RM}$ with $5 \mathrm{~min}$ of rest between sets and $30 \mathrm{sec}$ of $\mathrm{VH}(\sim 50$ breaths per minute) prior to the start of each set in well trained power athletes. This protocol was shown to effectively increase blood $\mathrm{pH}$ and lower $\mathrm{P}_{\mathrm{ETCO}} \mathrm{CO}_{2}$ to a range of $15-25 \mathrm{~mm} \mathrm{Hg}$ without significant adverse effects ${ }^{4,6,7}$. Additionally, $\mathrm{VH}$ resulted in a significant attenuation in velocity decrement from set to set ${ }^{7}$. Greater repetitions to failure lead to increases in overall training volume which has been shown to be favorable for muscular hypertrophy ${ }^{10}$, while the attenuation of velocity decrement across resistance training sets suggests a potential for greater power outputs during a workout. These implications are important for performance enhancement. However, it is unknown if similar results would occur in recreationally trained individuals or if VH results are similar using different training intensities and inter-set recovery times. Therefore, the purpose of this study was to investigate the effects of inter-set $\mathrm{VH}$ during $70 \%$ and $90 \% 1 \mathrm{RM}$ bench press (BP) and squat (SQ) exercise on repetitions to failure, power, and velocity in moderately trained individuals. A secondary aim was to investigate the impact of $\mathrm{VH}$ on muscle oxygen kinematics and blood lactate. Given the recent findings from Sakamoto et al. ${ }^{7}$ we hypothesized that $\mathrm{VH}$ would result in greater repetitions to failure and an attenuation in power and velocity decrement from set to set. Additionally, we hypothesized that $\mathrm{SmO} 2$ would differ between protocols and that $\mathrm{VH}$ would result in an attenuation of the typical rise in blood lactate.

\section{Scientific Methods}

Participants

Fifteen recreationally trained (at least 3 days/week of resistance training for at least 3 months) college-aged males (18 to 22 years) with an average of $2.92 \pm 2.18$ years of weightlifting experience volunteered for this study (Table 1). Subjects were excluded if they had any diagnosed cardiovascular, metabolic, or respiratory diseases, mental disorders, or major injuries that prevented them from participating in physical activity. Additionally, subjects were excluded if they were currently taking creatine, caffeine supplements, steroids, or other performance-enhancing drugs. Subjects were screened for eligibility using a health history questionnaire. All subjects were instructed to refrain from the use of any performance enhancing supplements/drugs throughout the duration of the study and were asked to maintain their normal training and nutritional habits. Additionally, subjects were asked to refrain from strenuous physical activity 24 hours prior to all testing sessions. Before enrolling, all subjects were informed of the possible risks and discomforts associated with participation prior to providing their written informed consent. All experimental procedures were approved by the university Institutional Review Board (IRB) prior to implementation.

Table 1: Demographic information (means \pm SD) for subjects $(n=15)$

\begin{tabular}{ll}
\hline Variable & Value \\
\hline Age (years) & $20.27 \pm 1.39$ \\
Height (cm) & $182.40 \pm 7.42$ \\
Mass (kg) & $82.23 \pm 10.84$ \\
BMI (kg/m $)$ & $24.65 \pm 2.28$ \\
Body Fat (\%) & $13.04 \pm 4.11$ \\
Fat Free Mass (kg) & $71.31 \pm 8.63$ \\
Fat Mass (kg) & $10.91 \pm 3.79$ \\
1RM Bench Press (kg) & $97.27 \pm 20.69$ \\
1RM Back Squat (kg) & $131.06 \pm 22.69$ \\
Training Experience (years) & $2.92 \pm 2.18$ \\
\hline
\end{tabular}

Protocol

A randomized crossover design was used to assess the impact of performing VH during rest between sets on reps to failure, average and peak bar velocity and power output, muscle oxygen saturation, and blood lactate in the BP and SQ 
exercises. Subjects performed a dynamic warmup followed by 3 sets of BP and SQ at $70 \%$ of their one repetition maximum (1RM) with 2 minutes of rest between sets and 3 sets of BP and SQ at 90\% 1RM with a 5 -minute inter-set rest period. During rest intervals, subjects breathed normally (CON) or performed VH during the last 30 seconds of their rest period. The order of conditions and training intensities were randomized, and sessions were separated by 48 72 hours. Total set repetitions, bar velocity, power output, and blood lactate were measured and recorded after each set. Session RPE (sRPE) was recorded at 5 minutes following the conclusion of each trial. Muscle oxygen saturation was measured continuously for the pectoralis major and the rectus femoris.

\section{Procedures}

Orientation Session and Maximal Strength Tests. During the first visit all experimental procedures and devices including determination of $1 \mathrm{RM}$ for the BP and SQ, the VH protocol, the OMNI RPE scale, blood lactate analyzer and the muscle oxygen saturation sensor were explained. Following the orientation, anthropometric measures were obtained including height $(\mathrm{cm})$, mass $(\mathrm{kg})$, fat free mass $(\mathrm{kg})$ and fat mass $(\%$ and $\mathrm{kg})$. Height $(\mathrm{cm})$ was measured using a physician's scale (Detecto, Webb City, MO). Weight (kg) and body composition (fat and lean mass) were measured using a Tanita bioelectrical impedance analyzer (BIA) (MC-980Uplus, Tanita Corporation of America, Arlington Heights, Illinois). Each subject then performed $30 \mathrm{sec}$ of the $\mathrm{VH}$-aided recovery breathing at a rate of 60 breaths per minute while following a metronome. Immediately following the VH familiarization, 1RMs for BP and SQ were determined using procedures established by NSCA ${ }^{11}$.

Experimental Sessions. During sessions 2-5 subjects completed a standardized dynamic warm-up followed by a workout consisting of 3 sets of BP and then SQ at either $70 \%$ or $90 \% 1 \mathrm{RM}$ using either VH or normal (CON) spontaneous breathing between sets. The intensity performed and breathing condition were randomly assigned using a random number generator (www.randomizer.org) and each session was separated by at least 48-72 hours 7 . During the 70\% 1RM session subjects completed 3 sets to failure of BP with 2 minutes of rest between sets and then a 5 -minute rest prior to performing 3 sets of SQ with 2 minutes of rest between sets. The $90 \% 1 \mathrm{RM}$ sessions were identical to the $70 \% 1 \mathrm{RM}$ sessions with 5 minutes of rest between all sets. For sessions in which VH was used, subjects breathed by following a metronome set at 60 beats per minute for the last 30 seconds of each inter-set rest period. During CON conditions subjects were instructed to breathe spontaneously for the entire inter-set rest period. Throughout each session subjects were verbally encouraged to perform as many repetitions as possible with proper technique until failure. Failure was defined as the inability to fully complete the concentric portion of each exercise.

Average and peak bar velocity $(\mathrm{m} / \mathrm{s}$ ) and power (W) were recorded using an accelerometer (PUSH Band 2.0, manufacturer, Toronto, Canada) attached to the left side of the barbell during both BP and SQ. Power output, bar velocity, and repetitions were measured automatically and stored by the Push Band phone application. Although the reliability and validity of the Push Band 2.0 is mixed, a recent study concluded it to be an accurate and reliable measurement of concentric velocity in both the BP and SQ ${ }^{12}$. Muscle oxygen saturation $\left(\mathrm{SmO}_{2}\right)$ was measured continuously throughout each session with a near-infrared spectroscopy (NIRS) device (Moxy Monitor, Hutchinson, Minnesota, US). Self-adhesive tape was used to affix the sensors to the upper pectoralis major before the bench press and the mid rectus femoris before the squat. Crum et al. ${ }^{13}$ determined the Moxy sensor to be a valid and reliable device for measuring SmO2. Blood lactate was measured immediately following each set of BP and SQ using capillary blood samples and a blood lactate analyzer (Lactate Plus, Nova Biomedical). Samples were collected using a lancet following cleaning of the fingertip with an alcohol swab and then drying. The first droplet was wiped away with a cotton swab to remove any alcohol and the subsequent droplets were used for analysis. Overall session rating of perceived exertion (sRPE) was collected using the Adult OMNI Resistance Exercise Scale ${ }^{14} 5$ min post completion of the final set of exercise.

\section{Statistical Analysis}

Statistical tests were performed using SPSS version 26.0 software (SPSS Inc., Chicago, IL). Statistical significance was set a priori at $p \leq 0.05$. Descriptive statistics were calculated for each variable and data were tested for normality using the Shapiro-Wilk test. A 2 (intensity: 70\% vs 90\%) x 2 (breathing condition: VH vs CON) repeated measures ANOVA was used to assess the effect of breathing intervention, intensity and interaction on total reps, mean power, velocity, SmO2, and blood lactate for BP and SQ and overall sRPE. A two (intensity) x two (breathing condition) x 3 (time: set 1 vs. set 2 vs. set 3) repeat measures ANOVA was used to assess the effect of intensity, breathing intervention, time and interactions on repetitions to failure, peak and mean power and velocity and blood lactate for each set of BP and SQ. Post hoc analyses of significant main and interaction effects were conducted using the Bonferroni adjustment to 
determine which conditions were significantly different. The assumption of sphericity was confirmed using Mauchly's test. Greenhouse-Geisser epsilon corrections were used when the sphericity assumption was violated.

\section{Results}

Total repetitions and repetitions to failure

There was no significant interaction between condition and intensity for total repetitions performed in the BP ( $p=$ $0.494)$ or $\mathrm{SQ}(p=0.448)$, nor were there any main effects for condition $(p=0.081 \& 0.305)$. A significant main effect for intensity was observed $\left(p^{\prime} s<0.001\right)$ showing that significantly more reps were completed during the $70 \%$ sessions than $90 \%$ sessions for BP and SQ (Table 2 and Figure 1).

There was no significant interaction between condition and intensity across each of the three sets for repetitions to failure in the BP or SQ $(p=0.963 \& 0.245)$. Additionally, there was no significant interaction between condition and sets $(p=0.726 \& 0.146)$ or between intensity and condition $(p=0.531 \& 0.462)$ for BP or SQ. There was a significant interaction between intensity and sets for both BP and SQ $\left(p^{\prime} s<0.001\right)$ showing a larger decrease in reps from set 1 to 2 at $70 \% 1 \mathrm{RM}$.

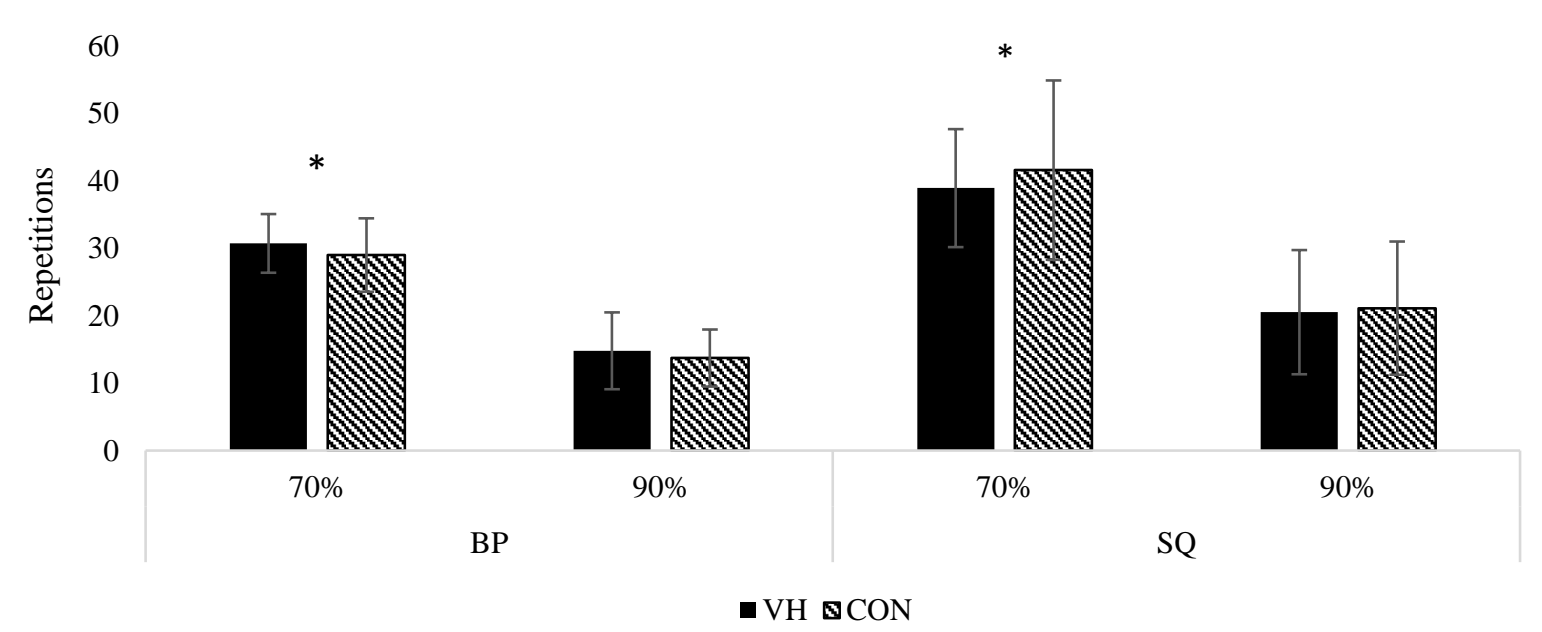

Figure 1: Total repetitions for BP and SQ at 70 and $90 \% 1 \mathrm{RM}$ with $\mathrm{VH}$ or $\mathrm{CON}$ condition. $\mathrm{BP}=$ bench press, SQ $=$ squat, $\mathrm{VH}=$ voluntary hyperventilation, $\mathrm{CON}=$ control. $* 70 \% 1 \mathrm{RM}$ significantly greater total repetitions than $90 \% 1 \mathrm{RM}$ for both BP and SQ (p's < 0.001)

Peak and mean power

There was no significant interaction between condition and intensity across each of the three sets for peak power in the BP or SQ $(p=0.497 \& 0.462)$. Additionally, there was no significant interaction between condition and sets $(p=$ $0.105 \& 0.415)$ or between intensity and condition $(p=0.273 \& 0.731)$ for BP or SQ. There was a significant interaction between intensity and sets for $\mathrm{BP}(p<0.001)$ showing a larger decrease in peak power from set 1 to 2 at $70 \% 1 \mathrm{RM}$. There was no significant interaction between intensity and sets $(p=0.182)$ or any main effects for intensity, condition, or sets $(p ' s>0.05)$ for SQ.

There was no significant interaction between condition and intensity across each of the three sets for mean power in the BP or SQ $(p=0.199 \& 0.301)$. Additionally, there was no significant interaction between condition and sets $(p=$ $0.086 \& 0.882)$ or between intensity and condition $(p=0.332 \& 0.890)$ for BP or SQ. There was a significant interaction between intensity and sets for both $\mathrm{BP}$ and SQ $\left(p^{\prime} s<0.001\right)$ showing an incremental decrease in mean power with each set at 70\% 1RM but an increase from set 1 to 2 at $90 \% 1 \mathrm{RM}$.

\section{Peak and mean bar velocity}

There was no significant interaction between condition and intensity across each of the three sets for peak velocity in the BP or SQ $(p=0.735 \& 0.329)$. Additionally, there was no significant interaction between condition and sets $(p=$ $0.134 \& 0.324)$ or between intensity and condition $(p=0.120 \& 0.568)$ for BP or SQ. There was a significant interaction between intensity and sets for $\mathrm{BP}(p<0.001)$ showing a larger decrease in peak velocity from set 1 to 2 at $70 \% 1 \mathrm{RM}$. There was no significant interaction between intensity and sets for SQ $(p=0.415)$ nor where there any main effects 
for condition or sets $(p ' s<0.05)$. There was a main effect for intensity $(p=0.021)$ showing that $70 \% 1 \mathrm{RM}$ resulted in higher peak velocity than 90\% 1RM for SQ.

There was no significant interaction between condition and intensity across each of the three sets for mean velocity in the BP or SQ $(p=0.225 \& 0.403)$. Additionally, there was no significant interaction between condition and sets $(p=$ $0.181 \& 0.512)$ or between intensity and condition $(p=0.096 \& 0.587)$ for BP or SQ. There was a significant interaction between intensity and sets for both BP and SQ ( $\not$ 's $<0.001)$ showing a larger decrease in mean velocity from set 1 to 2 at 70\% 1RM for BP. For SQ mean velocity decreased with each set at 70\% 1RM but increased from set 1 to 2 and then decreased from 2 to 3 at $90 \% 1 \mathrm{RM}$.

Mean $\mathrm{SmO}_{2}$

There was a significant interaction between condition and intensity for mean $\mathrm{SmO}_{2}$ of the pectoralis $(p=0.034)$ showing that $\mathrm{HV}$ resulted in a higher mean SmO2 at 90\% 1RM (78.26 \pm 12.02 vs. $71.30 \pm 13.03)$ but a lower mean $\mathrm{SmO}_{2}$ at $70 \% 1 \mathrm{RM}(72.32 \pm 14.63$ vs. $76.11 \pm 10.21)$ than CON. There was no significant interaction for mean $\mathrm{SmO} 2$ of the rectus femoris $(p=0.338)$ nor was there a main effect for condition $(p=0.714)$. There was a main effect for intensity $(p=0.043)$ showing that $\mathrm{SmO}_{2}$ was significantly higher at $90 \%$ than $70 \%$ (Table 2 ).

\section{Blood lactate}

There was no significant interaction between condition and intensity across each set for BL during BP or SQ $(p=$ $0.236 \& 0.808)$. There was no significant interaction between condition and intensity $(p=0.226 \& 0.591)$ or intensity and sets $(p=0.061 \& 0.709)$. There was a significant interaction between condition and sets during SQ $(p=0.037)$ (but not for BP $(p=0.316)$ showing an incremental increase in BL from one set to the next, however, VH attenuated the increase from set 2 to 3 ( +0.56 vs. $+1.08 \mathrm{mmol}$ at $70 \%$, $+0.25 \mathrm{vs} .+1.16 \mathrm{mmol}$ at $90 \%$ ). For BP significant main effects for sets $(p<0.001)$ and intensity $(p<0.001)$ were found. BL significantly increased from set 1 to 2 and from 2 to 3. BP at $70 \% 1 \mathrm{RM}$ resulted in significantly higher BL than $90 \% 1 \mathrm{RM}$. There was no main effect for condition for $\mathrm{BP}(p=0.568)$ (Figure 2).

\section{(A) Bench Press}

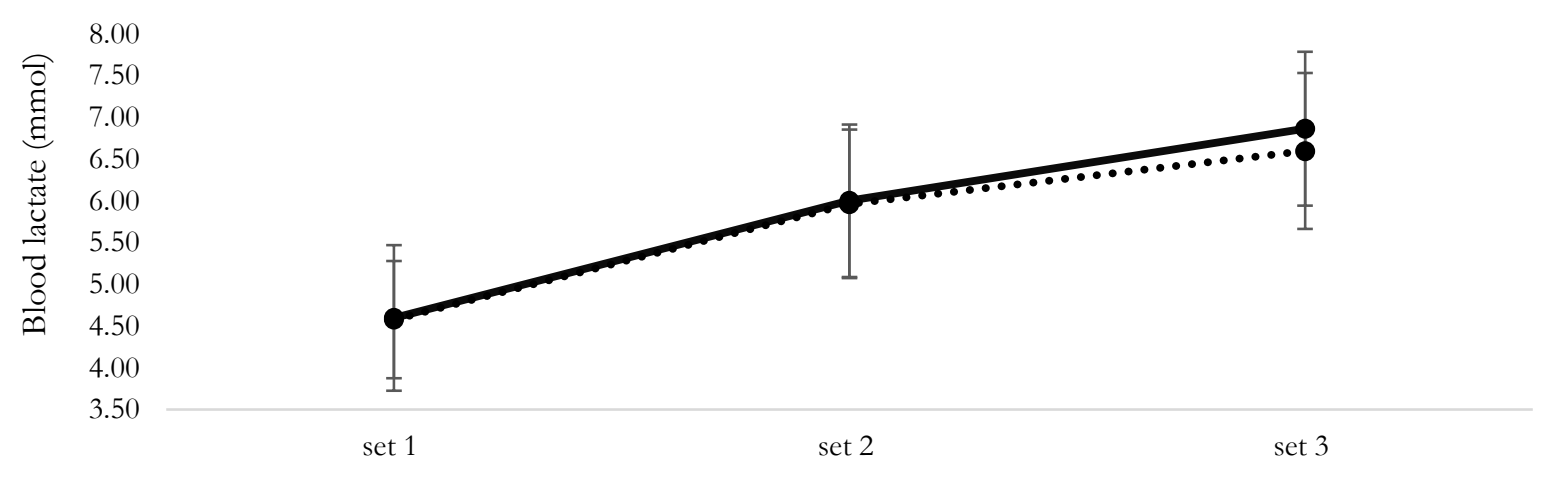

\section{(B) Squat}

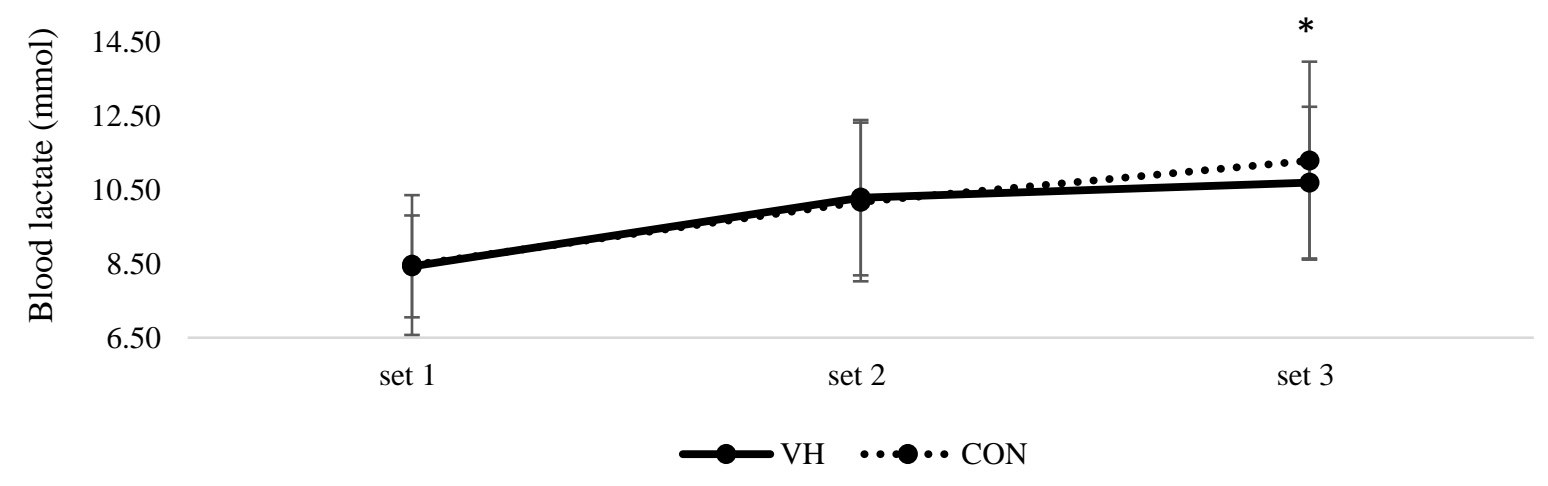


Figure 2. Blood lactate (data are mean \pm SD of $70 \& 90 \%$ intensities) across sets for Bench Press (A) and Squat (B). $\mathrm{VH}=$ voluntary hyperventilation, $\mathrm{CON}=$ control. $*$ significant interaction between condition and sets $(p=0.037)$. VH condition resulted in smaller increase in average blood lactate of both intensities from set 2 to 3 of SQ.

Session RPE

There was no significant interaction between condition and intensity for sRPE $(p=0.818)$. There was no significant main effect for condition ( $p=0.751)$ however, there was a significant main effect for intensity $(p=0.036)$ showing that sRPE was significantly higher at $70 \%$ than $90 \% 1 \mathrm{RM}$ (Table 2 ).

Table 2. Total repetitions, SmO2 and sRPE (means \pm SD) for BP and SQ at 70 and $90 \% 1 \mathrm{RM}$ in VH and CON conditions $(\mathrm{n}=15)$

\begin{tabular}{|c|c|c|c|}
\hline Variable & $70 \% 1 \mathrm{RM}$ & $90 \% 1 \mathrm{RM}$ & P-value, Effect Size \\
\hline Total Reps BP & & & intensity, $\mathrm{p}<0.001, \eta_{\mathrm{p}}{ }^{2}=0.952$ \\
\hline VH & $30.7 \pm 4.4^{*}$ & $14.8 \pm 5.7$ & condition, $\mathrm{p}=0.081,{\eta_{\mathrm{p}}}^{2}=0.034$ \\
\hline $\mathrm{CON}$ & $29.0 \pm 5.5^{*}$ & $13.7 \pm 4.2$ & interaction, $\mathrm{p}=0.494, \eta_{\mathrm{p}}{ }^{2}=0.201$ \\
\hline Total Reps SQ & & & intensity, $\mathrm{p}<0.001, \eta_{\mathrm{p}}^{2}=0.932$ \\
\hline VH & $38.9 \pm 8.8^{*}$ & $20.5 \pm 9.2$ & condition, $\mathrm{p}=0.305, \mathrm{\eta}_{\mathrm{p}}{ }^{2}=0.075$ \\
\hline CON & $41.6 \pm 13.3^{*}$ & $21.13 \pm 9.9$ & interaction, $\mathrm{p}=0.448, \eta_{\mathrm{p}}^{2}=0.042$ \\
\hline Average Power BP & & & intensity, $\mathrm{p}=0.021, \mathrm{\eta}_{\mathrm{p}}{ }^{2}=0.324$ \\
\hline VH & $279.43 \pm 60.34 *$ & $255.18 \pm 31.57$ & condition, $\mathrm{p}=0.407, \eta_{\mathrm{p}}^{2}=0.050$ \\
\hline CON & $275.46 \pm 55.92 *$ & $269.88 \pm 75.89$ & interaction, $\mathrm{p}=0.271, \eta_{\mathrm{p}}{ }^{2}=0.086$ \\
\hline Average Power SQ & & & intensity, $\mathrm{p}=0.015, \eta_{\mathrm{p}}{ }^{2}=0.352$ \\
\hline VH & $648.84 \pm 136.09 *$ & $604.74 \pm 105.15$ & condition, $\mathrm{p}=0.813, \mathrm{\eta}_{\mathrm{p}}{ }^{2}=0.004$ \\
\hline CON & $662.02 \pm 153.16^{*}$ & $599.96 \pm 114.32$ & interaction, $\mathrm{p}=0.557, \eta_{\mathrm{p}}^{2}=0.025$ \\
\hline Average Velocity BP & & & intensity, $\mathrm{p}<0.001, \eta_{\mathrm{p}}^{2}=0.938$ \\
\hline VH & $0.35 \pm 0.04 *$ & $0.26 \pm 0.04$ & condition, $\mathrm{p}=0.862, \eta_{\mathrm{p}}^{2}=0.002$ \\
\hline CON & $0.34 \pm 0.05^{*}$ & $0.27 \pm 0.05$ & interaction, $\mathrm{p}=0.065, \eta_{\mathrm{p}}{ }^{2}=0.222$ \\
\hline Average Velocity SQ & & & intensity, $\mathrm{p}=<0.001, \eta_{\mathrm{p}}{ }^{2}=0.850$ \\
\hline HV & $0.46 \pm 0.06^{*}$ & $0.36 \pm 0.05$ & condition, $\mathrm{p}=0.735,{\eta_{\mathrm{p}}}^{2}=0.008$ \\
\hline CON & $0.46 \pm 0.07 *$ & $0.36 \pm 0.05$ & interaction, $\mathrm{p}=0.823, \eta_{\mathrm{p}}^{2}=0.004$ \\
\hline Average SmO2 BP (\%) & & & intensity, $\mathrm{p}=0.829, \eta_{\mathrm{p}}{ }^{2}=0.003$ \\
\hline VH & $72.3 \pm 14.6$ & $78.3 \pm 12.0^{* *}$ & condition, $\mathrm{p}=0.615, \eta_{\mathrm{p}}{ }^{2}=0.019$ \\
\hline CON & $76.1 \pm 10.2 * *$ & $71.3 \pm 13.0$ & interaction, $\mathrm{p}=0.034, \eta_{\mathrm{p}}{ }^{2}=0.283$ \\
\hline Average SmO2 SQ (\%) & & & intensity, $\mathrm{p}=0.043, \eta_{\mathrm{p}}{ }^{2}=0.261$ \\
\hline VH & $41.7 \pm 16.2$ & $48.5 \pm 15.4^{*}$ & condition, $\mathrm{p}=0.714, \mathrm{\eta}_{\mathrm{p}}^{2}=0.010$ \\
\hline CON & $40.8 \pm 9.2$ & $51.9 \pm 18.4^{*}$ & interaction, $\mathrm{p}=0.338, \eta_{\mathrm{p}}{ }^{2}=0.0 .66$ \\
\hline Average Blood Lactate BP & & & intensity, $\mathrm{p}<0.001, \eta_{\mathrm{p}}{ }^{2}=0.625$ \\
\hline VH & $6.30 \pm 1.10^{*}$ & $5.34 \pm 1.08$ & condition, $\mathrm{p}=0.563, \eta_{\mathrm{p}}{ }^{2}=0.025$ \\
\hline CON & $6.41 \pm 0.93^{*}$ & $5.01 \pm 0.78$ & interaction, $\mathrm{p}=0.225, \eta_{\mathrm{p}}{ }^{2}=0.103$ \\
\hline Average Blood Lactate SQ & & & intensity, $\mathrm{p}<0.001, \eta_{\mathrm{p}}{ }^{2}=0.573$ \\
\hline VH & $11.25 \pm 1.87 *$ & $8.35 \pm 2.81$ & condition, $\mathrm{p}=0.330, \eta_{\mathrm{p}}{ }^{2}=0.068$ \\
\hline $\mathrm{CON}$ & $11.56 \pm 2.32 *$ & $8.67 \pm 2.42$ & interaction, $\mathrm{p}=0.977, \eta_{\mathrm{p}}{ }^{2}=0.001$ \\
\hline sRPE & & & intensity, $\mathrm{p}=0.036, \eta_{\mathrm{p}}^{2}=0.278$ \\
\hline VH & $9.1 \pm 0.5^{*}$ & $8.8 \pm 0.9$ & condition, $\mathrm{p}=0.751, \mathrm{\eta}_{\mathrm{p}}^{2}=0.007$ \\
\hline CON & $9.2 \pm 0.7 *$ & $8.8 \pm 0.9$ & interaction, $\mathrm{p}=0.818, \eta_{\mathrm{p}}^{2}=0.004$ \\
\hline
\end{tabular}

$\mathrm{SmO} 2=$ muscle oxygen saturation, $\mathrm{sRPE}=$ session rating of perceived exertion, $\mathrm{BP}=$ bench press, $\mathrm{SQ}=$ squat, $\mathrm{sRPE}=$ session rating of perceived duration, $\mathrm{VH}=$ voluntary hyperventilation, $\mathrm{CON}=$ control, $*$ significant $(p<$ 
$0.05)$ difference between intensities regardless of condition, ** significant $(p<0.05)$ interaction between intensity and condition

\section{Discussion}

The primary aim of this study was to investigate the effects of $\mathrm{VH}$ on repetitions to failure, bar velocity, and power output in BP and SQ at 70 and $90 \%$ of $1 \mathrm{RM}$. A secondary aim was to investigate the effects of VH on muscle oxygen saturation and blood lactate during exercise. Overall, repetitions to failure and peak and mean power and bar velocity decreased from set to set in BP and SQ during both training intensities and inter-set recovery conditions. The $70 \%$ $1 \mathrm{RM}$ sessions resulted in significantly more total repetitions completed, greater peak and mean power and velocity, blood lactate, and sRPE than the $90 \%$ 1RM sessions. Contrary to our initial hypothesis, VH did not mitigate the decrease in repetitions, power, or velocity from set to set, nor did it result in any significant difference in total repetitions or sRPE. As expected, blood lactate increased from set to set with each intensity, however, VH resulted in a slight attenuation of this increase from set 2 to 3 during SQ at both training intensities.

Our findings conflict with those of other investigations 3,4,6,7 in which more highly trained individuals using VH improved performance outcomes. Generally, at similar workloads more highly trained individuals display lower respiratory quotients and suppressed rates of glycogen depletion thereby delaying fatigue and prolonging physical exertion to a greater extent than less trained individuals ${ }^{15}$. The subjects in the current study had $2.92 \pm 2.18$ years of resistance training experience (2-3 days/week). In contrast, Sakamoto et al. ${ }^{7}$ used power-trained athletes with $6.8 \pm$ 3.1 years of resistance training experience and found significant positive effects for repetitions to failure, joint angular velocity, and power output using VH. Additionally, Sakamoto and colleagues ${ }^{4,6}$ found significant attenuations in performance decrements during repeated cycling sprints using $\mathrm{VH}$ with well-trained university athletes (having competed in their sport for nearly 7 years with training occurring 5-6 days/week). Furthermore, Jacob et al. ${ }^{3}$ recruited highly trained individuals with at least 5 years of training experience and found that average velocity had improved when performing a 50-meter swim front crawl after a VH protocol.

Despite such findings, the usefulness of $\mathrm{VH}$ as an ergogenic aid remains questionable with several studies finding no benefit to performance based outcomes ${ }^{8,9,16-18}$. Hilbert et al. ${ }^{18}$ found no performance benefits to repeated bouts of handgrip exercise following VH in untrained males. Similarly, Kaçoğlu and Işik's ${ }^{17}$ work resulted in inconclusive findings, with $\mathrm{VH}$ prior to activity improving 10 -m sprint times but showing no difference in countermovement jump of moderately trained young female volleyball players. Several other studies have reported no differences in peak or mean power achieved during cycling sprints but did not specifically note the training experience of their subjects $8,9,16$. Accordingly, these works suggest that other factors may contribute to the effectiveness of $\mathrm{VH}$ including timing and duration of $\mathrm{VH}$, rest time, and training experience to name a few.

Although controversial ${ }^{19}$, the accumulation of blood lactate has been considered a key element in the onset of fatigue during exercise (primarily due to the subsequent increase in $\mathrm{H}^{+}$ions) ${ }^{15}$. Research has consistently demonstrated that more highly trained individuals can clear blood lactate at significantly faster rates than less trained individuals ${ }^{20}$. In the present study the highest average blood lactate level of nearly $13 \mathrm{mmol} / \mathrm{L}$ was higher than the maximum level achieved in Sakamoto et al. ${ }^{7}(9.82 \mathrm{mmol} / \mathrm{L})$. It is possible that the lower training experience of our subjects led to reduced rates of lactate clearance and increased rates of lactate production (stemming from accelerated glycolysis), potentially too high to see an ergogenic effect from VH. Additionally, the present study utilized different training intensities and rest periods than Sakamoto et al. ${ }^{7}$ Specifically a slightly higher training intensity $(90 \% 1 \mathrm{RM})$ with a similar 5 -minute rest period, and a lower intensity $(70 \% 1 \mathrm{RM})$ with a much lower 2 -minute rest period was used. This suggests that the effectiveness of $\mathrm{VH}$ may be impacted by training intensity, duration of rest, and training experience.

We hypothesized that $\mathrm{VH}$ would result in an attenuation of the rise in blood lactate from one set to the next. Our results, however, only showed a slight attenuation of blood lactate accumulation from set 2 to 3 during the squat exercise, and this did not result in any performance differences. Chiappa et al. ${ }^{21,22}$ offer a potential suggestion for the attenuation seen in the present study, noting that the increased work of ventilatory muscles associated with $\mathrm{VH}$ may facilitate the uptake or re-utilization of blood lactate accumulated from exercise by those muscles. This claim, however, is controversial with several studies showing no buffering of blood lactate due to increased work from ventilatory muscles ${ }^{23,24}$. Additionally, it is possible that this increased work associated with VH may have neutralized the potential positive effects of $\mathrm{VH}$ by inducing greater fatigue and feelings of discomfort, especially given our subjects were less trained than those in other studies. According to Sakamoto et al. ${ }^{5}$ additional negative effects of VH include impaired 
hemodynamics, slower $\mathrm{O}_{2}$ kinetics, and reduced central motor command via reduced cerebrovascular blood flow. Research indicates that higher fitness levels generally result in improved hemodynamic responses, faster $\mathrm{O}_{2}$ kinetics and greater cerebrovascular blood flow than lower fitness levels ${ }^{25-27}$. Therefore, it is possible that these effects may have also played a role in neutralizing the potential ergogenic effects of $\mathrm{VH}$ in our subjects.

Another possible explanation for our results, especially with respect to the $70 \% 1 \mathrm{RM}$ training intensity, is that the recovery period used in this study ( 2 minutes) was simply not sufficient to allow the body's acid-base balance to restore. In 2015, Sakamoto et al. investigated the effects of VH on peak torque and EMG of vastus lateralis and medialis during repeat sets of isokinetic knee extension exercise at two different contraction speeds in well trained power athletes. The recovery period between sets was only $40 \mathrm{sec}$. Aside from a minor ergogenic effect on peak torque at 60 degrees/second, $\mathrm{VH}$ did not attenuate peak torque or EMG activity decrement from set to set ${ }^{5}$. The possible combined effects of a shorter rest period, greater work, and greater perceived effort (greater total reps, blood lactate accumulation, and sRPE) in the 70\% 1RM sessions with the lesser trained status of our subjects may provide another explanation for the null findings.

Controlling HV with minimal equipment (i.e., a metronome) is a practical application of similar intra-set recovery strategies used in prior research, however, a primary limitation of this study is that that $\mathrm{P}_{\mathrm{et}} \mathrm{CO}_{2}$ and $\mathrm{pH}$ levels were not measured during the $\mathrm{VH}$ protocol to ensure respiratory alkalosis. Although the protocol used in this study has been shown to lower $\mathrm{P}_{\mathrm{et}} \mathrm{CO}_{2}$ and elevate $\mathrm{pH}^{7}$ in highly trained subjects it is possible that our subjects did not reach the desired level of $\mathrm{P}_{\mathrm{et}} \mathrm{CO}_{2}(15-25 \mathrm{~mm} \mathrm{Hg})$ to realize an ergogenic effect. This warrants further investigation of $\mathrm{HV}$-aided recovery protocols with lesser trained individuals in which parameters indicating respiratory alkalosis are directly measured.

\section{Conclusion}

Voluntary hyperventilation is the act of intentionally increasing the depth and rate of one's breathing resulting in respiratory alkalosis (increase in $\mathrm{pH}$ ). Recently, $\mathrm{VH}$ has been used as a pre-activity or intra-set recovery strategy to improve performance or reduce performance decrements during repeated bouts of physical activity in well-trained individuals. In the present study, however, $\mathrm{VH}$ aided recovery resulted in no significant differences in total repetitions or set to set decreases in reps, power or barbell velocity in the bench press or squat when performed at 70 and $90 \%$ 1RM in recreationally trained individuals. Although VH did provide a small but significant attenuation of blood lactate accumulation during the squat exercise it is important to note that respiratory alkalosis was not directly measured, making it difficult to infer any cause-and-effect relationships without further investigations. In the context of the current literature regarding $\mathrm{VH}$, our findings suggest that the effectiveness of $\mathrm{VH}$ may be influenced by many factors including one's training experience.

\section{Acknowledgements}

None

\section{Conflict of Interest}

No conflict of interest

\section{References}

1. Chin LMK, Leigh RJ, Heigenhauser GJF, Rossiter HB, Paterson DH, Kowalchuk JM. Hyperventilation-induced hypocapnic alkalosis slows the adaptation of pulmonary $\mathrm{O} 2$ uptake during the transition to moderate-intensity exercise. J Physiol. 2007;583(1):351-364. doi:10.1113/jphysiol.2007.132837

2. Gardner WN. The pathophysiology of hyperventilation disorders. CHEST. 1996;109(2):516-534. doi:10.1378/chest.109.2.516

3. Jacob C, Keyrouz C, Bideau N, et al. Pre-exercise hyperventilation can significantly increase performance in the 50-meter front crawl. Sci Sports. 2015;30. doi:10.1016/j.scispo.2015.02.006

4. Sakamoto A, Naito H, Chow C. Hyperventilation as a strategy for improved repeated sprint performance. J Sci Med Sport. 2013;16:e44. doi:10.1016/j.jsams.2013.10.106

5. Sakamoto A, Naito H, Chow CM. Hyperventilation-induced respiratory alkalosis falls short of countering fatigue during repeated maximal isokinetic contractions. EurJ Appl Physiol. 2015;115(7):1453-1465. doi:10.1007/s00421015-3134-8 
6. Sakamoto A, Naito H, Chow CM. Effects of hyperventilation on repeated pedaling sprint performance: Short vs. long intervention duration. J Strength Cond Res. 2018;32(1). https://journals.lww.com/nscajscr/Fulltext/2018/01000/Effects_of_Hyperventilation_on_Repeated_Pedaling.20.aspx

7. Sakamoto A, Naito H, Chow CM. Hyperventilation-aided recovery for extra repetitions on bench press and leg press. J Strength Cond Res. 2020;34(5). https://journals.lww.com/nscajscr/Fulltext/2020/05000/Hyperventilation_Aided_Recovery_for_Extra.10.aspx

8. Fujii N, Tsuchiya SI, Tsuji B, Watanabe K, Sasaki Y, Nishiyasu T. Effect of voluntary hypocapnic hyperventilation on the metabolic response during Wingate anaerobic test. Eur J Appl Physiol. 2015;115. doi:10.1007/s00421-015-3179-8

9. Dobashi K, Fujii N, Ichinose M, Fujimoto T, Nishiyasu T. Voluntary hypocapnic hyperventilation lasting 5 min and 20 min similarly reduce aerobic metabolism without affecting power outputs during Wingate anaerobic test. Eur J Sport Sci. Published online August 19, 2020:1-8. doi:10.1080/17461391.2020.1812728

10. Schoenfeld BJ, Contreras B, Krieger J, et al. Resistance Training Volume Enhances Muscle Hypertrophy but Not Strength in Trained Men. Med Sci Sports Exerc. 2019;51(1):94-103. doi:10.1249/MSS.00000000000001764

11. McGuigan M. Administration, Scoring, and Interpretation of Selected Tests. In: Essentials of Strength and Conditioning. 4th ed. Human Kinetics; :259-316.

12. Pelka E, Williams A, McLaughlin D, Gadola C, Slattery E, Claytor R. Comparison of concentric movement velocity with push band 2.0 and vicon motion capture during resistance exercises. Int Soc Biomech Sport. 2020;38(1):252-255.

13. Crum EM, O'Connor WJ, Van Loo L, Valckx M, Stannard SR. Validity and reliability of the Moxy oxygen monitor during incremental cycling exercise. Eur J Sport Sci. 2017;17(8):1037-1043. doi:10.1080/17461391.2017.1330899

14. Robertson R, Goss F, Rutkowski J, et al. Concurrent validation of the OMNI perceived exertion scale for resistance exercise. Med Sci Sports Exerc. 2003;35 2:333-341.

15. Fitts R. Mechanisms of muscular fatigue. In: Principles of Exercise Biochemistry. Vol 46. 3rd ed. Karger; 2004:279300.

16. Morrow JA, Fell RD, Gladden LB. Respiratory alkalosis: no effect on blood lactate decline or exercise performance. Eur J Appl Physiol. 1988;58(1):175-181. doi:10.1007/BF00636623

17. Kaçoğlu C, Işık M. The acute effects of the voluntary pre-activity hyperventilation on jump and sprint performance in female volleyball players. Eur J Hum Mov. 2017;2017:93-104.

18. Hilbert M, Shushakov V, Maassen N. The influence of respiratory acid-base changes on muscle performance and excitability of the sarcolemma during strenuous intermittent hand grip exercise. $J$ Appl Physiol. 2011;112(4):571-579. doi:10.1152/japplphysiol.00869.2010

19. Brooks GA. Lactate doesn't necessarily cause fatigue: why are we surprised? J Physiol. 2001;536(Pt 1):1-1. doi:10.1111/j.1469-7793.2001.t01-1-00001.x

20. Gmada N, Bouhlel E, I M, et al. Effect of combined active recovery from supramaximal exercise on blood lactate disappearance in trained and untrained man. Int. J. Sports Med. 2005; 26: 1-6. Int J Sports Med. 2005;26:16. doi:10.1055/s-2005-837464

21. Chiappa GR, Roseguini BT, Alves CN, Ferlin EL, Neder JA, Ribeiro JP. Blood lactate during recovery from intense exercise: impact of inspiratory loading. Med Sci Sports Exerc. 2008;40(1):111-116. doi:10.1249/mss.0b013e3181591de1

22. Chiappa G, Ribeiro J, Alves C, et al. Inspiratory resistive loading after all-out exercise improves subsequent performance. Eur J Appl Physiol. 2009;106:297-303. doi:10.1007/s00421-009-1022-9

23. Brown PI, Sharpe G, Johnson MA. Loading of trained inspiratory muscles speeds lactate recovery kinetics. Med Sci Sports Exerc. 2010;42 6:1103-1112.

24. Johnson M, Mills D, Brown D, Bayfield K, Gonzalez J, Sharpe G. Inspiratory loading intensity does not influence lactate clearance during recovery. Med Sci Sports Exerc. 2011;44:863-871. doi:10.1249/MSS.0b013e31824079d0

25. Hamer M. The effects of exercise on haemodynamic function in relation to the familial hypertension risk model. J Hum Hypertens. 2006;20(5):313-319. doi:10.1038/sj.jhh.1001999

26. Marwood S, Roche D, Rowland T, Garrard M, Unnithan V. Faster pulmonary oxygen uptake kinetics in trained versus untrained male adolescents. Med Sci Sports Exerc. 2010;42 1:127-134.

27. Smith EC, Pizzey FK, Askew CD, et al. Effects of cardiorespiratory fitness and exercise training on cerebrovascular blood flow and reactivity: a systematic review with meta-analyses. Am J Physiol-Heart Circ Physiol. 2021;321(1):H59-H76. doi:10.1152/ajpheart.00880.2020 
